\title{
Vertical MEMS Resonators for Real-Time Clock Applications
}

\author{
A. Pomarico, ${ }^{1}$ A. Morea, ${ }^{1}$ P. Flora, ${ }^{1}$ G. Roselli, ${ }^{1}$ and E. Lasalandra ${ }^{2}$ \\ ${ }^{1}$ STMicroelectronics, Via Arnesano Km 5 clo Distretto Tecnologico ISUFI, Palazzina B, 73100 Lecce, Italy \\ ${ }^{2}$ STMicroelectronics, Via Tolomeo 120010 Cornaredo, MI, Italy
}

Correspondence should be addressed to A. Pomarico, anna.pomarico@st.com

Received 20 July 2010; Accepted 15 October 2010

Academic Editor: P. Siciliano

Copyright ( 2010 A. Pomarico et al. This is an open access article distributed under the Creative Commons Attribution License, which permits unrestricted use, distribution, and reproduction in any medium, provided the original work is properly cited.

\begin{abstract}
MEMS resonators are today widely investigated as a desirable alternative to quartz resonators in real-time clock applications, because of their low-cost, integration capability properties. Nevertheless, MEMS resonators performances are still not competitive, especially in terms of frequency stability and device equivalent resistance (and, then, power consumption). We propose a new structure for a MEMS resonator, with a vertical-like transduction mechanism, which exhibits promising features. The vertical resonator can be fabricated with the low-cost, high performance THELMA technology, and it is designed to be efficiently frequency tunable. With respect to the commonly investigated lateral resonators, it is expected to have lower equivalent resistances and improved large-scale repeatability characteristics.
\end{abstract}

\section{Introduction}

Oscillators have a very important role in modern electronics. Frequency references are in fact required in every clocked electronic system, including communication circuits, microprocessors, and samples signal processing circuits. The typical operating frequency that is generally used in timecontrol applications is $32.768 \mathrm{kHz}$.

Nowadays, oscillators in commercial devices consist of high performance piezoelectric crystals, in particular quartz oscillators. The advantages of the crystal oscillators are that their frequency is very stable and their quality factor is extremely high. The major disadvantages are the crystal dimensions and the impossibility to integrate the crystal itself with the electronics.

From the point of view of microelectronics, there is a strong boost to the development of silicon, fully integrated, oscillators. Integration is important not only for size shrinkage but also for economic purposes and power reduction requirements. MEMS (micro electro-mechanical Systems) technology allows this. It is possible to realize an integrated silicon oscillator using the mechanical properties of silicon devices.

A MEMS resonator is a micromachined mechanical structure that exhibits a resonant behavior. The device is driven by a sinusoidal signal at a drive electrode, and the resulting output is read out from a sense electrode. The target specifications for commercial MEMS resonators are dictated by the system-level requirements, which are low phase noise, low power consumption, and reduced frequencytemperature drift. The micromechanical resonator must then have high quality factor, low equivalent resistance, and high intrinsic frequency stability versus temperature or, should that not be the case, high tuning capabilities.

The frequency stability versus temperature is one of the fundamental challenges in the design and fabrication of a mechanical silicon resonator. In fact, while piezoelectric resonators are intrinsically quite stable respect to the temperature variations, MEMS resonators are very sensitive to them, due to the fact that the Young modulus of silicon has relevant temperature dependence. In uncompensated MEMS resonators, the temperature-related frequency drift over the usual operation range $\left(-40 \div 85^{\circ} \mathrm{C}\right)$ is typically of the order of $10 \mathrm{ppm} /{ }^{\circ} \mathrm{C}$ to $20 \mathrm{ppm} /{ }^{\circ} \mathrm{C}$, corresponding to an overall frequency variation of up to about $2500 \mathrm{ppm}$ [1]. This leads to the need of specific design/technological strategies to maintain the temperature steadiness of the frequency. One of the most effective strategies is to design MEMS resonators whose operating frequency is dependent on the polarization voltage applied across the resonatorto-electrode gap, so that the frequency can be easily electrically tuned to compensate any temperature-induced variations. 


\section{Theoretical Background: Electromechanical Transduction in MEMS Resonators}

A key design point for MEMS resonators is how to perform the electromechanical transduction of the energy from the mechanical excitation to the electrical signal and vice versa. For the $32 \mathrm{kHz}$ resonators, two kinds of electromechanical coupling between the oscillating mass and the driving and sensing electrodes are possible at this aim: the "comb-finger" type coupling and the "parallel-plate" type coupling. They both are today exploited in lateral-type MEMS resonators, in which the vibration of the micromachined movable mass occurs in a plane parallel to the silicon surface. In the combfinger resonators [2] the signal between the driving electrode, the movable mass- and the sensing electrode is transmitted by a comb-finger coupling between interdigitated electrodes. Using a lumped equivalent parameters model, it can be demonstrated that the equivalent resistance has the following expression:

$$
R_{\mathrm{eq}}=\frac{k_{\mathrm{eq}} d^{2}}{\omega Q 4 V_{P}^{2} N^{2} \varepsilon^{2} h^{2}},
$$

where $k_{\text {eq }}$ is the equivalent spring constant of the resonator, $d$ is the transduction gap (distance between adjacent fingers), $N$ is the number of fingers, $\varepsilon$ is the permeability of the air and $h$ is the overlap of fingers. In this kind of structure, the reduction of the gap value and/or the increase of the number of fingers are the elective strategies to reduce the equivalent resistance and to increase the electromechanical coefficient. The main disadvantage of comb-finger resonators is that the frequency is not dependent, at least to the first order, on the DC polarization voltage $V_{p}$, applied across the resonatorto-electrode gap. Only the presence of nonidealities such as levitation and end effects (fringing fields) introduces some electric-dependent component of the spring constant and then some variation of frequency with varying $V_{p}$ although, the variations that can be induced are too small to effectively compensate the temperature-dependent frequency shifts.

The parallel-plate coupling [3] overcomes some disadvantages of the comb-fingers resonator. In the parallelplate coupling the signal between the driving electrode, the movable mass and the sensing electrode is transmitted like in a parallel plate capacitor. The reduction of the equivalent resistance, when decreasing the gap size, is more efficient than in the comb-drive structures, due to its dependence on the fourth power of the gap size. In fact, in a lumped equivalent parameters formulation, the equivalent resistance of a MEMS resonator having a parallel-plate-type transduction has the following expression:

$$
R_{\mathrm{eq}}=\frac{k_{\mathrm{eq}} d^{4}}{\omega Q V_{P}^{2} \varepsilon^{2} A^{2}},
$$

where $k_{\text {eq }}$ is the equivalent spring constant of the resonator, $d$ is the transduction gap (distance between faced electrodes), $\omega$ is the angular frequency, $Q$ is the quality factor, $V_{p}$ is the polarization voltage applied to the resonator, $\varepsilon$ is the permeability of the air, and $A$ is the area of faced electrodes.
A very interesting feature of parallel-plate-type devices is that the frequency is voltage dependent. It can be calculated that the frequency tuning coefficient has the following expression:

$$
\frac{\Delta f}{f_{0}}=\frac{\varepsilon A}{k_{\mathrm{eq}} d^{3}} V_{P}^{2} .
$$

The frequency is then easily controllable by adjusting $V_{p}$, and temperature compensation can be performed. This is a key issue for a commercial MEMS resonator. We then decided to adopt the parallel-plate transduction model in the design of MEMS resonators.

\section{Technological Challenges in MEMS Resonators Fabrication}

MEMS resonators fabrication entails the use of micromachining techniques able to provide high-quality structural layers and small lateral transduction gaps, as these are fundamental points in order to get high-quality factors and low equivalent resistances.

For general MEMS devices fabrication, a very advantageous technology proved to be the THELMA (tHick epitaxial layer for microgyroscopes and accelerometers) process [4]. THELMA is a high-performance, low-cost technology consisting of a proprietary combination of manufacturing steps of bulk and surface micromachining technologies. One of its more distinctive features is that the thick structural layer is patterned by dry anisotropic etching, which provides vertical walls of etched trenches ( $89^{\circ}$ over $15 \mu \mathrm{m}$ layer).

Structures designed in THELMA technology have only two silicon layers: the structural layer (epitaxial polysilicon) and a thin polysilicon layer used for anchoring and biasing of epitaxial layer structures. These two layers are separated from each other and from the substrate by two oxide layers. The structure of a device in THELMA technology and its main characteristics are shown in Figure 1 and in Table 1.

Considering the specific case of MEMS resonators, their fabrication in THELMA technology is highly desirable because it ensures a competitive cost and some remarkable device characteristics, namely, the good mechanical properties of epitaxial polysilicon, which translate into low mechanical losses and then favorably affects the quality factor of resonators. Yet, there are still some technological open issues that need to be solved for the fabrication of highperformance MEMS resonators in THELMA technology.

Traditional MEMS resonators are based on lateral transduction geometry. In this kind of resonators, any attempts to reduce the equivalent resistance and increase the tuning efficiency meet two main technological limitations. The first one is the relatively small size of the coupling area between the movable mass and the electrode, as one dimension of the coupling area is necessarily limited by the structural layer thickness, which is $15 \mu \mathrm{m}$ in THELMA technology. The second one is represented by the technological limits in reducing the value of the lateral gap. In the case of THELMA structures, the minimum, experimental lateral gap between two adjacent features is about $2.3 \mu \mathrm{m}$. This is a medium 


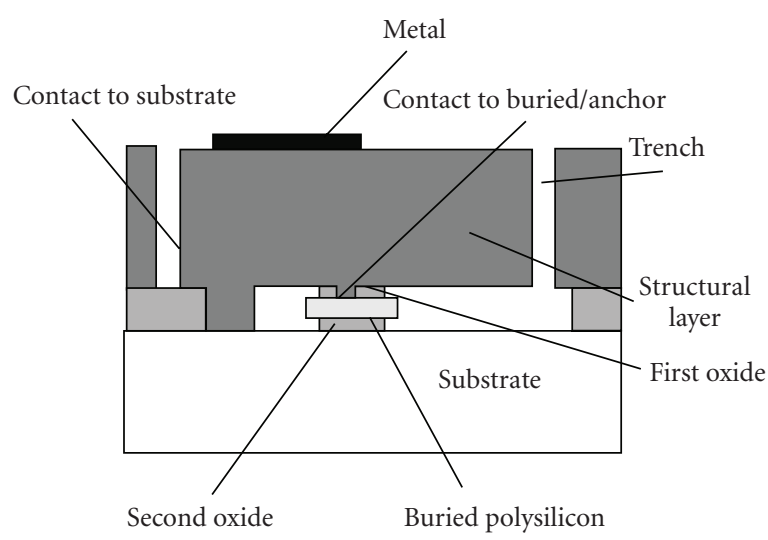

FIgure 1: Structure of a device in THELMA technology.

TABLE 1: Thelma technology parameters.

\begin{tabular}{lccccc}
\hline & $\begin{array}{c}\text { Structural } \\
\text { layer }\end{array}$ & $\begin{array}{c}\text { 1st } \\
\text { oxide }\end{array}$ & $\begin{array}{c}\text { Buried poly } \\
\text { silicon }\end{array}$ & $\begin{array}{c}\text { 2nd } \\
\text { oxide }\end{array}$ & Substrate \\
\hline $\begin{array}{l}\text { Thickness, } \\
\mu \mathrm{m}\end{array}$ & 15 & 1.6 & 0.45 & 2.5 & - \\
Resistance & $10 \Omega /$ square & - & $25 \Omega /$ square & - & $1 \Omega / \mathrm{cm}$ \\
\hline
\end{tabular}

value in fact the lateral gap is actually affected by the technological spreads of the etching process. A $2.3 \mu \mathrm{m}$ lateral gap would correspond, for typical values of vacuum pressure $(0.2 \mathrm{mbar})$ and of lateral coupling areas, to an equivalent resistance of tens of $M \Omega$. Such a resistance is unacceptable because the control circuit should apply a current of tens of volts with consequent very high power consumption. Actually, in lateral resonators only very small transduction gaps (order of magnitude of $1 \mu \mathrm{m}$ or hundreds of $\mathrm{nm}$ ) would give the possibility to obtain equivalent resistances of the order of a few hundreds $\mathrm{k} \Omega$ or less, as required for market purposes. In order to attain small lateral gaps, some researchers addressed expensive MEMS technologies, such as the HARPSS (high aspect-ratio combined polyand single-crystal silicon micromachining) technology [5]. Nevertheless, the use of such high-cost processes would reduce MEMS resonators competitiveness.

\section{Vertical MEMS Resonators}

The solution that we want to propose, in order to solve these problems, is to introduce a new design strategy for MEMS resonators. Our leading idea was to keep the use of the high-performance, low-cost THELMA technology, because we wanted to maintain its advantages and to try to overcome its gap-size and coupling-area limitations by addressing a new concept of MEMS resonator, that we called "vertical resonator" [6]. In vertical resonators the coupling is parallelplate type and the oscillation occurs along $z$ direction (that is the normal to the silicon wafer surface) instead of in $x-y$ plane (lateral resonators).

In Figure $2(\mathrm{a}, \mathrm{b})$ a schematic of a vertical resonator is reported.
In THELMA standard technology this design is preferable because the gap along the $z$ direction, formed by the sacrificial oxide layer removal, is typically smaller $(1.8 \mu \mathrm{m})$. Moreover, its value is repeatable with a higher precision level, which can have a favorable impact on the large scale production.

In our vertical resonators, the bottom electrode has the same lateral shape of the shuttle and is a little larger than it. In this way, the coupling area is represented by the whole $x-y$ surface of the shuttle. As $32 \mathrm{kHz}$ operation frequency corresponds to shuttles having lateral sizes of a few hundreds microns, coupling area for vertical resonators is typically much larger than that for lateral resonators, so improving the electromechanical coupling, with positive consequences on the reduction of the equivalent resistance and on the tuning coefficient improvement. This aspect is made more relevant by the fact that, as finite element method (FEM) simulations demonstrated, we can design shuttle and spring beams in such a way that the shuttle always stays parallel to the substrate during the vibration.

The distinctive features of the described vertical resonators, namely, their smaller transduction gap and their larger coupling area with respect to lateral resonators, are expected to translate into a reduced equivalent resistance, an improved electromechanical coupling coefficient, and an improved tuning coefficient. In short, this would denote a better fit of the market resonators specifications with respect to the state-of-the-art lateral resonators.

In Figure 3 we report a scanning electron microscope (SEM) image of an example of the vertical resonators we fabricated.

We also investigated different geometrical configurations for the vertical resonators, varying the shuttle shape and size, the number, length, and position of the springs, and the design of the spring folding. In the design, a special attention has to be dedicated to the springs number, position, and shape (length, width, and the possible presence of folds), as they are the main parameters influencing the equivalent spring constant. We also point out that, with a suitable design of spring beams, it is possible to increase or to decrease the area of the shuttle mass, keeping a constant value of the resonant frequency. It is then possible to choose within a range of equivalent spring constants and corresponding values for the shuttle area, which gives a high flexibility to the resonator device design and facilitates the achievement of the desired electromechanical performances. Finally, a suitable design of the spring beams can also ensure that in-plane $(x-y)$ vibration modes are far enough from the vertical fundamental vibration mode, that we want to exploit in such a structure, so avoiding the risk of noxious interferences.

According to theoretical evaluations, vertical resonators are expected to be characterized by remarkable $Q$ (quality factor) values, even in medium-vacuum conditions. As an example, preliminary theoretical estimation based on the boundary integral equation (BIE) formulation [7] resulted in a $Q$ factor of about 3000 for the vertical resonator shown in Figure 2, when operated at $0.2 \mathrm{mbar}$ pressure (prof A. Frangi, private communication). 


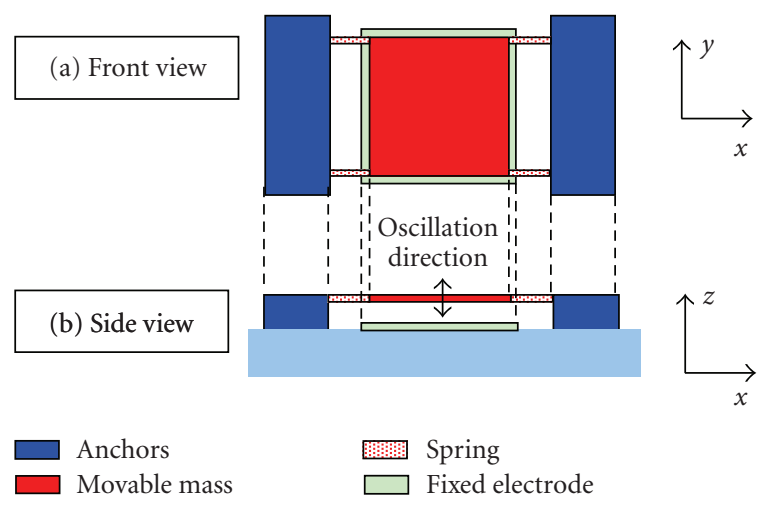

FIGURE 2: vertical resonator schematic draw: (a) front view; (b) side view.

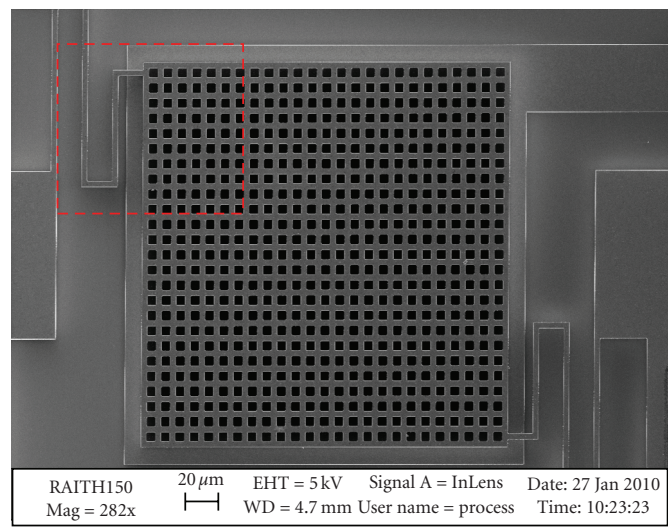

(a)

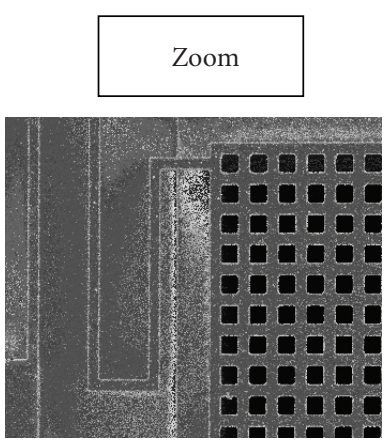

(b)

FIGURE 3: Example of implementation of a vertical resonator designed to have $32 \mathrm{kHz}$ natural frequency. The square is $454 \mu \mathrm{m}$ (side). The shuttle mass has release holes, having a square shape, with $5 \mu \mathrm{m} \times 5 \mu \mathrm{m}$ size and $4 \mu \mathrm{m}$ reciprocal distance.

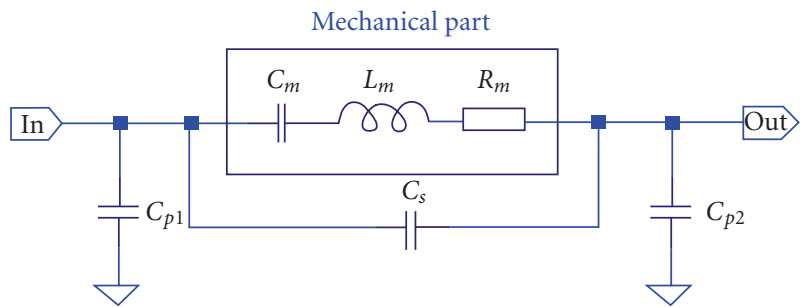

FIGURE 4: Equivalent circuit.

We theoretically analyzed the electrical response of vertical resonators by the use of a simplified electro-mechanical model, based on lumped equivalent parameters (Figure 4).

Assuming that $Q=3000$, as indicated by theoretical calculations, we analyzed the changes in the resonator response when varying operation conditions. In Figures 5 and 6 we report, respectively, the equivalent resistance and the resonant frequency versus the polarization voltage $V_{p}$.

Both the equivalent resistance and the frequency reduce when increasing the bias voltage. As shown in Figure 5, the application of just a few volts $(\geq 4 \mathrm{~V})$ of bias voltage is enough to get equivalent resistances of the order of a

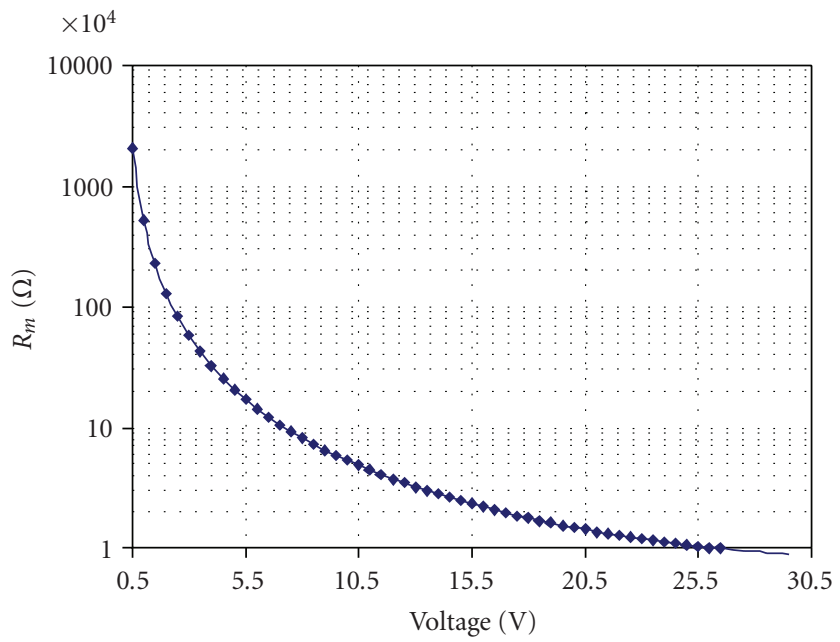

FIgURE 5: Equivalent resistance versus the polarization voltage $V_{p}$.

few hundreds $\mathrm{k} \Omega$ or less. Figure 6 proves that the frequency drift versus the bias voltage is quite relevant, so making the device efficiently frequency tunable. In particular, we expect 


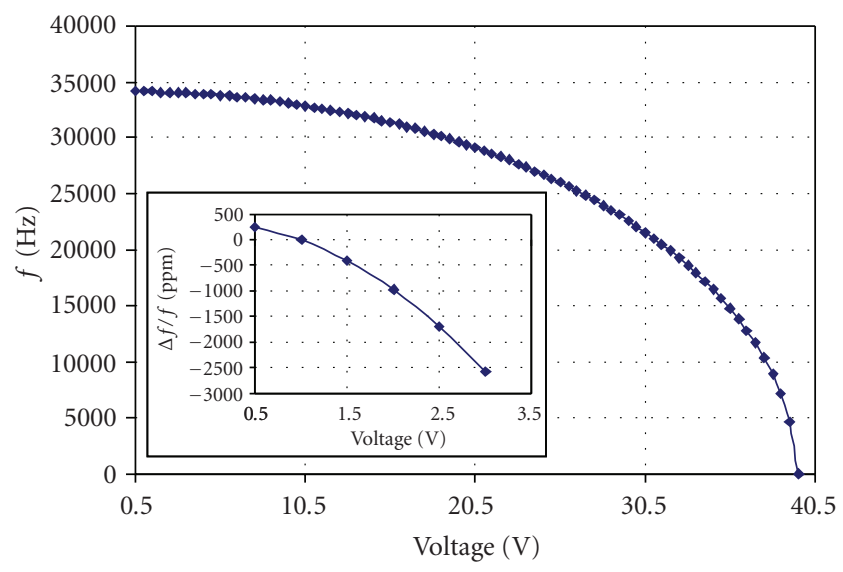

Figure 6: Frequency versus the polarization voltage $V_{p}$. inset: variations (expressed in parts per million or ppm) of the frequency with respect to its value at $V_{p}=1 \mathrm{~V}$, versus $V_{p}$.

that just small changes of the bias potential will be able to compensate the temperature- related frequency drift over the whole operation temperature range $\left(-40 \div 85^{\circ} \mathrm{C}\right)$. In order to further illustrate this point, let us assume that in our device the target frequency is obtained, in ambient conditions, for $1 \mathrm{~V}$ bias application (corresponding to $34100 \mathrm{~Hz}$ ). Moreover we assume that the overall frequency drift due to the temperature variations in the operation range is $2500 \mathrm{ppm}$ (typical value for MEMS resonators). Plotting the frequency variations (in ppm) as a function of the bias voltage (inset of Figure 6), we see that varying the voltage from $0.5 \mathrm{~V}$ to $3 \mathrm{~V}$ will correspond to a frequency variation of more than $2500 \mathrm{ppm}$. Then the variation of the bias voltage in a range of about $3.5 \mathrm{~V}$ should be enough to compensate the overall frequency drift due to the temperature variations.

\section{Conclusions}

In conclusion, we designed, theoretically investigated, and fabricated a new type of MEMS resonator, which differs from the state-of-the-art lateral resonators in its "verticallike" transduction mechanism. Our vertical resonator can be fabricated using the THELMA technology, a low-cost technology that is able to ensure very good electromechanical properties of the microengineered structures. This resonator gets three main advantages from the exploitation of the vertical gap, instead of the lateral gap: (1) the gap value can be established with a better accuracy, so increasing the reproducibility of the electromechanical characteristics of the device on a large scale; (2) the smaller value of the gap and the larger value of the coupling area reduce the equivalent resistance, which in turns reduces the power consumption combined with an active circuit implemented by using essentially a single transconductance device, such as MOS transistor in week inversion; and (3) the smaller value of the gap and the typical very large value of the shuttle area also improve the tunability coefficient, so making frequency tuning of the device significantly more efficient with respect to the lateral MEMS resonators.

\section{Acknowledgments}

The authors thank professor Frangi (Department of Structural Engineering, Politecnico di Milano) for the BIE model calculations of $Q$-values reported in this work.

\section{References}

[1] E. P. Quevy and R. T. Howe, US007211926B2 patent, 2007.

[2] W. C. Tang, T.-C. H. Nguyen, and R. T. Howe, "Laterally driven polysilicon resonant microstructures," Sensors and Actuators, vol. 20, no. 1-2, pp. 25-32, 1989.

[3] G.K.-F. Ho and K. Ayazi, US 2008/0186109 A1.

[4] C. Combi and B. Vigna, "Test structures for process flow control (Thelma process)," Tech. Rep., Sensors and Microactuators Group-STMicroelectronics, 1999.

[5] F. Ayazi and K. Najafi, "High aspect-ratio combined poly and single-crystal silicon (HARPSS) MEMS technology," Journal of Microelectromechanical Systems, vol. 9, no. 3, pp. 288-294, 2000.

[6] A. Pomarico, P. Flora, A. Morea, and G. Roselli, "Microelectromechanical resonant structure having improved electrical characteristics," patent pending, 2009.

[7] A. Frangi, A. Ghisi, and L. Coronato, "On a deterministic approach for the evaluation of gas damping in inertial MEMS in the free-molecule regime," Sensors and Actuators. A, vol. 149, no. 1, pp. 21-28, 2009. 

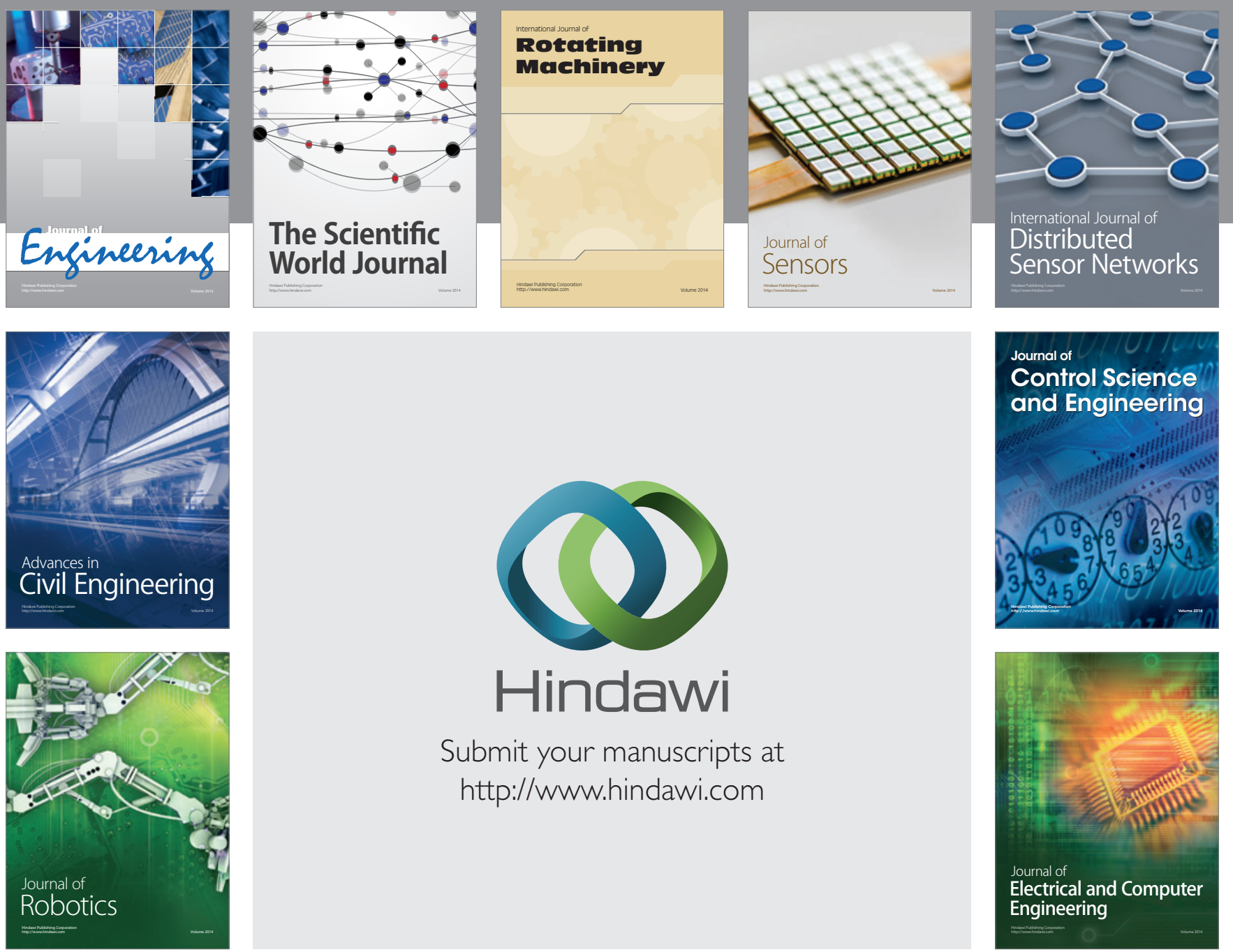

Submit your manuscripts at

http://www.hindawi.com
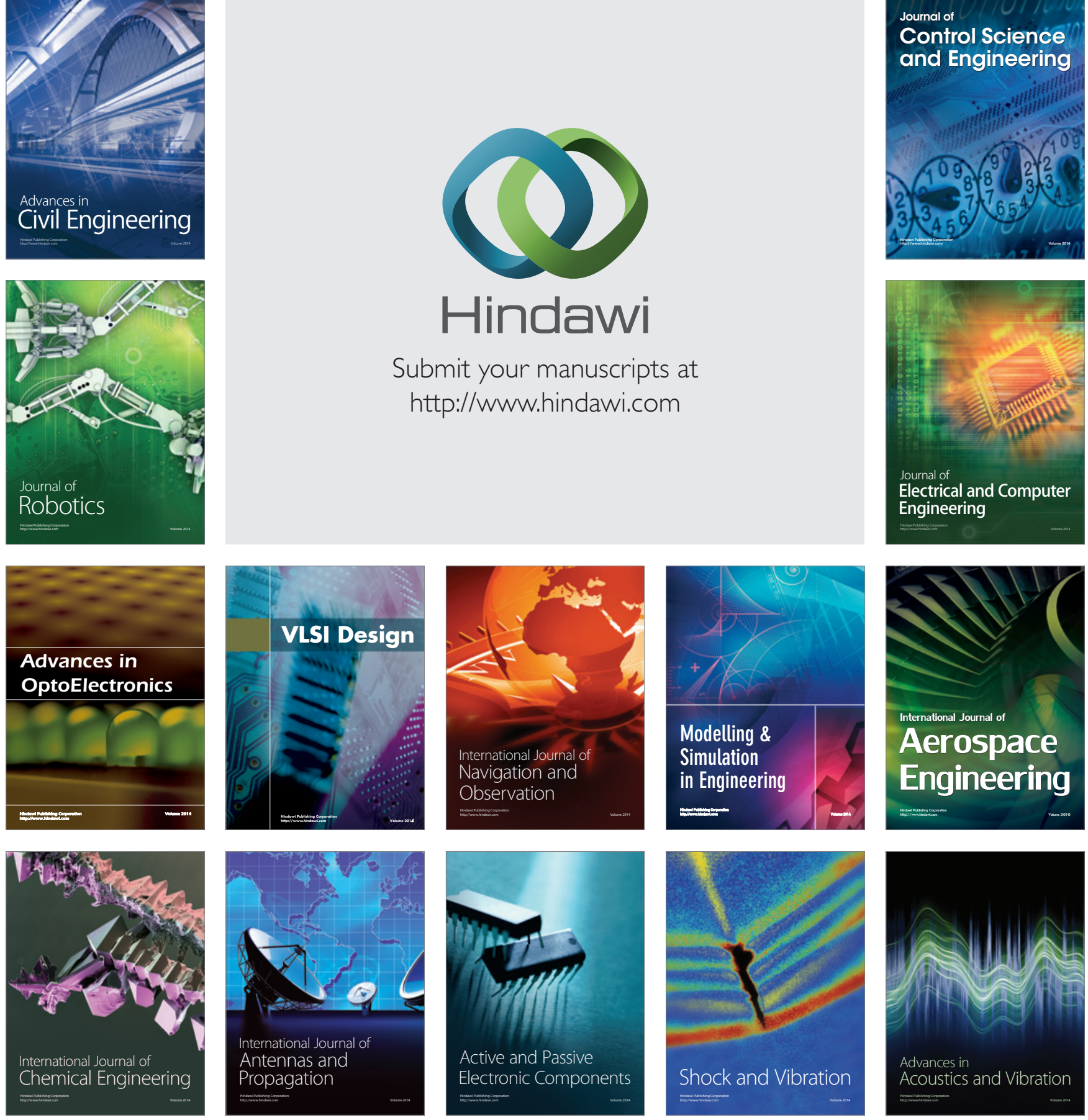